\title{
Design of agricultural machinery upon reliability indices
}

\author{
Yuriy Manshin ${ }^{1}$, Elena Manshina ${ }^{1, *}$ \\ ${ }^{1}$ Don State Technical University, Rostov-on-Don, Russian Federation
}

\begin{abstract}
Introduction. The formation of an algorithm for designing mechanical systems - machines in terms of reliability is considered. The algorithm is built with account for the latest publications on the standardization of reliability indices of machines and components at the initial stages of the project. The recovery cost technique, free from the need to use the exponential law when distributing reliability indices between system elements, is presented. The concepts of the required and achieved reliability factors and methods for obtaining them at different stages of the project are introduced. The possibilities of the project modification to provide a given level of reliability are considered. Research objective is to combine modern scientific developments on the standardization of reliability indices of machines, components, and methods for obtaining the required operation life with the design drafting stages.
\end{abstract}

Intuitive notion of the machine reliability in the early stages of the project comprehension under the development of technical specifications (TS) should be replaced by the parameters of the machine reliability principles as rendered in GOST 27.002-89 "Industrial product dependability. General concepts. Terms and definitions".

Longevity can be chosen on the basis of industry concepts and norms on the timing of the obsolescence of technology. Then the calendar service life of $T_{K}$, expressed in years, will determine the maximum running hours:

$$
T_{H}=T_{k} \cdot 365 \cdot K_{\text {год }} \cdot 24 \cdot K_{c y m} \cdot \Pi B, h,
$$

where $K_{\text {год }}$ is the coefficient of possible machine utilization per year; $K_{c y m}$ is the utilization factor $C$ per day; $\Pi B$ is the duty factor which is an average ratio of the on-time (acceleration time and steady-state time) to the time of the working cycle of the machine or its mechanisms.

The operating time $T_{H}$ can be a guideline when selecting overhaul periods and the operating time $t=T, h$, for which reliability characterized by the probability of survival $P(t)$ (POS), or reliability function, can be guaranteed.

* Corresponding author: elemans@mail.ru 
Depending on the designated area of the machine and operating techniques, repairability and serviceability characteristics may be added.

The adopted reliability parameters of the entire machine at the subsequent stages of the project should serve as the basis for developing a reliability system throughout the project structure.

Among the four aspects of the concept of "reliability", failsafety, in our opinion, is its basic property. This is undoubtedly the case in projects of nonrestorable technical systems (TS) and products. Overhaul periods of uptime form the longevity of the restorable TS. In various reliability tasks, a technical object can be considered as restorable (during the service life $T_{K}$ ) or as nonrestorable in the operational period of time (scheduled flight, terminable contract work, terminal seasonal farm work, etc.), when failsafety is the key and the only property of reliability [1]. Thus, the TS should contain the value of the POS $P(t)$, and the operating time $t=T, h$, for which the POS is guaranteed.

The operating time $t$ is an argument of the reliability function $P(t)$. For all kinds of reliability laws, $P(t)$ decreases monotonically with growing $t$. Thereby, to obtain the required POS, the operating time should be rationally minimized and selected in accordance with the machine behavior.

The project POS can be selected from the existing industry standards, from competitive conditions, according to a plan of improving product quality, and other considerations. High reliability (POS approaching $P(t)=1$ ) is possible under reducing the required operating time. In [2], the amount of operating time, within which it is possible to maintain the safety margin at $P(t)=1$, is validated. If necessary to significantly increase the operating time, high failsafety is supported by an increase in the cost of materials and technology, an increase in the costs of organizing a product quality assurance system [3; 4], an increase in operating costs (acceleration of maintenance of various volumes). A guideline for selecting a new design project for POS can be given by a table of conditional reliability levels adopted for engineering products. In [5], ideas about reliability levels were generalized according to a number of sources $[1,2,6]$, and a scope of categories was formed in several groups of the TS characteristics.

After choosing the required lifelength of $t=T$, $h$, and the required POS, $P(T)$, the exponential law of the system reliability in the following form:

$$
P(t)=e^{-\lambda t},
$$

provides the connection of failure rate $\lambda$ during normal operation after run-in with an average time between failures $\check{T}$ of the system:

$$
\lambda=1 / \breve{T}
$$

with the reliability parameters

$$
\lambda=(-\ln P(T)) / T
$$

from which the TS mean life can be calculated

$$
\widehat{T}=-T /(\ln P(T)) .
$$


If the initial time is the mean time between failures $\check{T}$ hours, according to the test results of a batch of machines, the law (2) provides the determination of the failure rate (4) and the corresponding POS TS (3).

\section{Structural Analysis}

In scientific sources and educational literature on reliability, the structural interelement coupling of the TS reliability is generally accepted as a bottom-up circuit analysis from POS elements to POS systems with sequential, concurrent and combined interaction of elements whose block schemes are widely known.

Under the sequential interaction of TS elements whose failures are independent, the exponential law (2) provides a simple mathematical model of reliability: if POS of the system $P(t)$ and the elements - subsystems $P_{u}(t)$ are in the ratio

$$
P(t)=\prod_{i=1}^{n} P_{i}(t),
$$

then the failure rates of the system $\lambda$ and subsystems $\lambda_{i}$ are in the ratio:

$$
\lambda=\sum_{i=1}^{n} \lambda_{i}
$$

The hierarchical block scheme formed from the nested levels of subsystems and elements $[1,7]$ permits to perform a top-down analysis from the TS POS to the POS of all elements. In this case, the failure rates (7) of the subsystems and elements are ranked depending on the accepted principle of the distribution of intensities. This reliability analysis corresponds to the sequence of design stages according to the USDD. The required values of the POS elements obtained at the stage of the preliminary design, and the required operating time are the initial data for the design of elements with a given level of reliability $[8,9]$ at the stage of the technical project.

However, a top-down analysis based on the law (2), which is used to describe POS of the systems in case of sudden failures, is valid, for example, for radio-electronic devices [10], whose structural elements are very complex products - subsystems of the lower level. But at the level of components of a mechanical system (MS) [11], degradation failures due to metal fatigue, wear, corrosion, and aging of materials are not described by the exponential law (2), hereat its application in these cases is incorrect.

Probably, for this reason, the papers present structural diagrams of machines to the level of subsystems, aggregates. But the POS of the MS assembly does not provide the basis for the design of its components with POS, which provides a given level of the machine reliability. And the development of a structural diagram of the TS reliability is advisable precisely for this purpose.

A bottom-up analysis of the TS POS with the concurrent interaction of $m$ elements is performed according to the known dependence between the probability of failure-free operation of the system through the POS of elements: 


$$
P(t)=1-\prod_{j=1}^{m}\left[1-P_{j}(t)\right]
$$

or for homogeneous elements:

$$
P(t)=1-\left[1-P_{j}(t)\right]^{m}
$$

are valid under the following conditions: the elements are switched on constantly, their failures are independent, each of the $m$ elements has POS $P_{j}(t)$ and is able to accept the input action and convert it into the output TS parameter. Then, the TS failure will occur after the failure of the last functional element.

With the known POS of systems and elements from (11), the number of elements $m$ can be obtained to ensure a given TS POS:

$$
m=\ln (1-P(t)) / \ln \left(1-P_{j}(t)\right)
$$

Application of the exponential law expression (2) does not provide simple options for a top-down circuit analysis, both for the relations $(10,11)$ and for more complex structures with concurrent interaction of elements (with switches, element redundancy and circuits, etc.). Perhaps for this reason, examples of a top-down circuit analysis of such structures are not given in the literature.

At the same time, it should be noted that a typical structure of machines is combined in nature, where a series of sequentially interacting blocks contains one or more blocks with concurrent interaction (redundancy) of the elements.

Complex structures with multidirectional interaction of elements having dependent failures, do not give simple possibilities of separation into elements. The functionality and reliability parameters should be determined for the entire system, which may entail a large amount of analytical and experimental work.

Graphic images of such schemes [2] are provided with mentioning about complexity of the functioning model and awkwardness of the reliability calculations (for example, complex closed interdependent operation of ICE units or a jet engine).

The objective of this paper is to build a design algorithm for TS - machines with specified reliability parameters. The algorithm should contain the following steps:

- selection of reliability parameters of durability, TS POS and the required operating time at the stage of TS;

- development of a hierarchical structural diagram of machine reliability at the stage of an engineering proposal;

- analysis of the structural diagram at all levels including the levels of elements at the stage of conceptual design;

- verification calculations of components for strength and endurance, calculations of the lifetime of the dynamically loaded components achieved in the project, standard and nonrepairable products at the stage of a technical project;

- assessment of the results and development of constructive, technological and organizational measures to bring the achieved lifetime closer to the required operating time.

In [5], a method for a top-down analysis of structural diagrams of machine reliability based on an alternative representation and ranking of the numerical value of POS $P(t)=$ $P(T)$, or probability of the failure $F(t)=F(T)$ of the TS within the boundaries of $0<P(T)$ 
$<1$, under the selected required operating time $t=T, h$, was developed and tested using examples to overcome the above contradictions under normalizing POS of the TS elements.

For sequentially interacting structures, the numerical value of the TS POS is presented by the power expression:

$$
P(T)=10^{X},
$$

from where the ranking parameter (RP) of the TS POS is

$$
X=\lg P(t)
$$

The numerical value of the TS POS through the POS of the elements is represented by the expression: $P(T)=P_{1}(T) * P_{2}(T) * \ldots * P_{n}(T)$, where $P_{1}(T) \ldots * P_{n}(T)$ are the numerical values of the POS of the elements also represented by $P_{i}(T)=10^{X i}$, where $X_{i}$ are the ranking parameters.

Parameters for ranking subsystems and TS elements:

$$
X_{i}=X a_{i}
$$

weight coefficient of the recovery value of sequentially interacting elements:

$$
\mathrm{a}_{\mathrm{i}}=\frac{\frac{1}{C_{i}}}{\sum \frac{1}{C_{i}}}
$$

where $C_{i}$ is the cost of recovering failed subsystems or elements.

For interacting concurrent structures, the numerical value of the probability of failure (PF) of the TS is presented by analogy with (14) through the power expression:

$$
F(T)=10^{Y},
$$

from where the ranking parameter of TS PF is

$$
Y=\lg F(T) .
$$

The numerical value of TS PF through PF of the elements is represented by the expression: $F(T)=\prod_{j=1}^{m} F_{j}(T)$, where the numerical value of $\mathrm{PF}$ of the elements are also represented by $F_{j}(T)=10^{Y_{j}}$, where $Y_{j}$ are ranking parameters $F_{j}(T)$.

The ranking parameter of the TS elements is

$$
Y_{j}=Y b_{j} .
$$


weight coefficient of the recovery value of interacting concurrent elements:

$$
\mathrm{b}_{\mathrm{j}}=\frac{C_{j}}{\sum C_{j}}
$$

where $C_{j}$ are recovery values of the failed components.

$$
\text { POS of the elements is } P_{j}(T)=1-F_{j}(T),
$$$$
\text { TS POS is } P(T)=1-\prod\left(1-P_{j}(T)\right) \text {. }
$$

The method algorithm coincides with the sequence of design stages: from the POS of the machine to the POS of its components. The selection of the required operating time and the distribution of POS throughout the components at the initial stages of the project provide designing with a given level of reliability at the subsequent stages. To universalize the approach to the formation of structural reliability schemes for various types of the element interaction, a structural scheme can be built on the basis of the construction structure. The criterion for ranking POS of the elements is the recovery value of the element in case of its failure. The recovery value of a failed element at any level of the structural scheme includes the costs of materials and products of the failed structural unit, elements that received secondary dependent failures, repair work, damage from the failure, in money equivalent. These costs are known with sufficient accuracy at the initial stage of machine development.

The need to use the exponential law when normalizing reliability and developing its structural schemes, is eliminated. The analysis of POS and PF is based only on the fundamental properties of reliability common to all technical objects and systems. The analysis algorithm is maintained at all levels of the structural schemes including levels of the components.

Mathematical models of structural schemes are simple, accessible for calculations at the early stages of the project, and equally effective for sequentially interacting, redundant, and combined reliability schemes. They provide convenient algorithms, programming and data correction capabilities.

The action of the method is considered on the numerical examples of the analysis of the reliability parameters of sequential, concurrent and combined circuits given in [5].

The application of the recovery value technique under the analysis of a multi-level structural scheme is considered in the example [5] of a top-down analysis of the structural scheme of a combine harvester.

After the required operating time is selected and the required POS of the components loaded with dynamic forces and moments are calculated, it is necessary to conduct a checking life prediction for them.

In [7-9], the methodology and algorithms for performing test calculations and refinement of parameters including the basic actions are given:

- using a statistical model of fatigue damage accumulation;

- determination of the median life of components based on the adjusted model of accumulation of fatigue damage: 


$$
N_{\sigma 0.5}=\frac{a_{\sigma} \sigma_{-1 д}{ }^{m_{\sigma}} N_{\mathrm{Б}_{\sigma}}}{\sum \sigma_{j}^{m_{\sigma}} p_{\sigma_{j}}}, \text { cycles }
$$

where $a_{\sigma}$ is the correction function; $\sigma_{-1 д}$ is shaft endurance limit according to the working draft of the technical design; $\sum \sigma_{j}^{m_{\sigma}} p_{\sigma_{j}}$ is a statistical model of fatigue damage accumulation;

- calculation of the average lifetime:

$$
t_{0.5}=\frac{N_{0.5}}{3600 n_{e}}, h,
$$

where $n_{e}, H z$, is average frequency of the effective amplitudes $(\sigma j \geq \sigma-1 \partial)$ involved in the process of fatigue damage accumulation;

- determination of the lifetime of components with the required POS according to the structural scheme, on the basis of a probabilistic model of the development of fatigue cracks represented by a logarithmically normal law, according to which the probability of failure-free operation expressed through the tabulated Laplace function is

$$
P(t)=\Phi\left(\frac{\lg t_{0.5}-\lg t}{S_{\lg t_{0.5}}}\right) .
$$

Here, $t$ is lifetime, $h$, corresponding to a given probability $P(t)$ of uptime; $t_{0.5}$ is the median life, $h$, corresponding to the probability of failure-free operation $P(t)=0.5 ; S_{\lg t_{0,5}}$ is standard deviation of the logarithm of the median life:

$$
S_{\lg t_{0.5}}=0,434 m \sqrt{V_{\sigma_{\text {-1д }}}^{2}+V_{\sigma_{j}}^{2}}
$$

where $V_{\sigma_{\text {-д }}}$ is the coefficient of variation of the endurance of the component, it can vary in the range of $0.05 \div 0.2 ; V_{\sigma_{j}}$ is the coefficient of variation of voltage amplitudes

The argument of Laplace function (25) is

$$
\frac{\lg t_{0.5}-\lg t}{S_{\lg t_{0.5}}}=u_{\mathrm{p}}
$$

where $u_{\mathrm{p}}$ is the fractile of normal distribution calculated in mathematical tables in the function $P(t)$ of the probability of failure-free operation.

From the formula (26), the lifetime distribution function is obtained in the form given in [12], which is graphically rectified in the logarithmic probability coordinates: 


$$
\lg t=\lg t_{0.5}-u_{p} S_{\lg t} . .5
$$

After solving the equation (27) with respect to $t$ given in $[9,10]$, we obtain the lifetime distribution function depending on the given POS:

$$
t=t_{0,5} 10^{-u P S \lg t_{0.5}}, h
$$

Lifetime $t$ can be interpreted as the lifetime $t_{\text {дос }}$ achieved in the project, which may be sufficient for $\mathrm{t} \geq \mathrm{T}$ or insufficient for $\mathrm{t}<\mathrm{T}$.

In the latter case, measures are needed to increase the achieved lifetime up to $t_{\text {пов. }}$. It follows from the function of the median life (23) that the lifetime can be enhanced either through increasing the endurance parameters or by reducing the stress amplitudes. For these tasks, there are many constructive procedures and techniques, whose verification according to the algorithm (23-28) represents a large amount of calculations. To reduce time on selecting reasonable options for modernization of the component, tools are developed [7-9] for an approximate assessment of the measures applied, resulting from the equality of lifetime ratios in hours and numbers of cycles to failure:

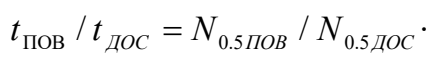

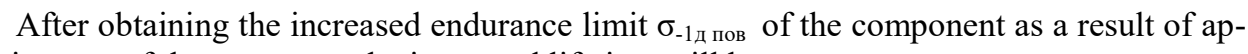
plying one of the measures, the increased lifetime will be:

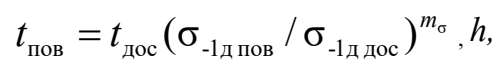

With a decrease in stress amplitudes through reducing the bending moment from $M_{\text {дос }}$ to $M_{\mathrm{yM}}$, we have:

$$
t_{\text {пов }}=t_{\text {дос }}\left(M_{\text {дос }} / M_{\text {ум }}\right)^{m_{\sigma}}
$$

If, for the same purpose, we increase the moment of resistance from the initial (achieved) value of $W_{\text {хдос }}$ to $W_{\text {хпов }}$, then we obtain:

$$
t_{\text {пов }}=t_{\text {дос }}\left(W_{x \text { пов }} / W_{x \text { дос }}\right)^{m_{\sigma}}
$$

With an increase in the shaft diameter from $d_{\text {дос }}$ to $d_{\text {пов }}$, we have:

$$
t_{\text {пов }}=t_{\text {дос }}\left(d_{\text {пов }} / d_{\text {дос }}\right)^{3 m_{\sigma}},
$$

where a high exponent of the ratio (33) at small increments of the shaft diameter causes a significant increase in the life of the component.

If at the first or next stage of modernization $t_{\text {пов }} \geq T$ is reached, the task of designing the component with the required level of reliability can be considered completed.

When assessing the divergence of values of the lifetime obtained from the function (28) and from approximate relations (30-33), which was performed by the numerical experiment $[8,9]$, a deviation range of $20 \ldots 100 \%$ of approximate lifetime upward from the functional ones was obtained. When comparing these deviations to scatter of the experimental 
results under endurance testing of components (up to 1000\% [12]), the divergence between the approximate and functional estimates of the lifetime is considered acceptable.

\section{Conclusion}

As a result of the solution to the problem, an algorithm for designing TS - machines with the specified reliability parameters is constructed. The algorithm provides a top-down analysis of the hierarchical structural diagram of the machine reliability, the result of which may be the required POS of all elements, ranked upon their recovery value in the event of failure. Using the laws of reliability of elements, the achieved lifetime can be brought up to standard, which is accelerated through using approximate estimates of the lifetime.

\section{References}

1. Yu.P. Manshin, E.Yu. Manshina, Interagromash, 169-171 (2014)

2. V.S. Viktorova, A.S. Stepanyants, LENAND, 256 (2016)

3. S.I. Malafeev, A.I. Kopeikin, Deer, 320 (2016)

4. V.Yu. Shishmarev, Academy, 304 (2010)

5. Yu.P. Manshin, E.Yu. Manshina, Vestnik of Don Technical University, 18 (4), 392-400 (2018)

6. G. N. Cherkesov, BKhV-Peterburg, 480 (2012)

7. Yu.P. Manshin, E.Yu. Manshina, Interagromash, 261-263 (2017)

8. Yu.P. Manshin, E.Yu. Manshina, Vestnik Mashinostroeniya, 12, 20-24 (2017)

9. Yu.P. Manshin, E.Yu. Manshina, Russian Engineering Research, 38 (3), 157-162 (2018)

10. S.I. Malafeev, LAN, 368 (2018)

11. I.A. Khozyaev, Monograph: Ministry of Education and Science of the Russian Federation, 235 (2014)

12. E.A. Lisunov, Textbook for universities. Special literature, 240 (2015) 